\title{
Translation, cross-cultural adaptation and psychometric validation of the Thai version of the STarT Back Screening Tool in patients with non-specific low back pain
}

Taweewat Wiangkham ${ }^{1,2^{*}}$ (D), Nattawan Phungwattanakul ${ }^{2}$, Natthathida Thongbai ${ }^{2}$, Nisa Situy ${ }^{2}$, Titipa Polchaika ${ }^{2}$, Isara Kongmee ${ }^{3}$, Duangporn Thongnoi ${ }^{3}$, Rujirat Chaisang ${ }^{3}$ and Wanisara Suwanmongkhon ${ }^{4}$

\begin{abstract}
Background: Low back pain (LBP) is a top musculoskeletal problem and a substantial cause of socioeconomic burden internationally. The STarT Back Screening Tool (SBST) is a useful screening tool to manage patients with LBP but it is unavailable in Thai. Therefore, the aims of this study were to translate and cross-culturally adapt the SBST into a Thai version (SBST-TH) and validate its psychometric properties (e.g., factor analysis, internal consistency, testretest reliability, agreement, convergent validity and discriminative validity).

Methods: Translation and cross-cultural adaptation of the SBST into Thai version were conducted according to standard guidelines. A total of 200 participants with non-specific LBP were invited to complete the SBST, visual analogue scale for pain intensity, Roland-Morris disability questionnaire (RMDQ), fear-avoidance beliefs questionnaire, pain catastrophising scale, hospital anxiety and depression scale and the EuroQol five-dimensional questionnaire. Thirty participants completed the SBST-TH twice with an interval of $48 \mathrm{~h}$ to evaluate test-retest reliability.
\end{abstract}

Results: Factor analysis demonstrated two (physical and psychological) components for the SBST-TH (39.38\% of the total variance). The Cronbach's alpha ( 0.86 for total score and 0.76 for psychosocial subscore) represent satisfactory internal consistency. The acceptability of intraclass correlation coefficient was found in the total (0.73) and subscore (0.79). The areas under the curve (AUC) for the total score ranged 0.67-0.85 and 0.66-0.75 for subscore. The excellent discriminative validity was observed ( $A \cup C=0.85,95 \%$ confidence interval $=0.72,0.97$ ) between the total score of the SBST-TH and disability (RMDQ). Spearman's correlation coefficients represented moderate to strong correlation (0.32-0.56) between the SBST-TH and all questionnaires. The findings suggest a good relationship between the SBST-TH and disability and quality of life. Owing to the results from the convergent and discriminative validity, construct validity of the SBST-TH can be supported. The minimal detectable changes of the total score and subscore were 2.04 and 1.60, respectively. Significant floor and ceiling effects were not found in the SBST-TH.

\footnotetext{
* Correspondence: taweewatw@nu.ac.th

${ }^{1}$ Exercise and Rehabilitation Sciences Research Unit, Faculty of Allied Health Sciences, Naresuan University, Phitsanulok 65000, Thailand

${ }^{2}$ Department of Physical Therapy, Faculty of Allied Health Sciences, Naresuan University, Phitsanulok 65000, Thailand

Full list of author information is available at the end of the article
}

(c) The Author(s). 2021 Open Access This article is licensed under a Creative Commons Attribution 4.0 International License, which permits use, sharing, adaptation, distribution and reproduction in any medium or format, as long as you give appropriate credit to the original author(s) and the source, provide a link to the Creative Commons licence, and indicate if changes were made. The images or other third party material in this article are included in the article's Creative Commons licence, unless indicated otherwise in a credit line to the material. If material is not included in the article's Creative Commons licence and your intended use is not permitted by statutory regulation or exceeds the permitted use, you will need to obtain permission directly from the copyright holder. To view a copy of this licence, visit http://creativecommons.org/licenses/by/4.0/ The Creative Commons Public Domain Dedication waiver (http://creativecommons.org/publicdomain/zero/1.0/) applies to the data made available in this article, unless otherwise stated in a credit line to the data. 
Conclusion: The SBST-TH was successfully translated and adapted. It is a valid and reliable tool to classify Thai patients with non-specific LBP into low, moderate and high risks for chronicity.

Trial registration: TCTR20191009005.

Keywords: Low back pain, Cross-cultural adaptation, Psychometric properties, STarT Back screening tool-Thai version, SBST-TH

\section{Background}

Low back pain (LBP) is a top musculoskeletal problem internationally [1] and a substantial cause for disability [2], especially non-specific LBP [3]. Individuals with non-specific LBP can experience pain, functional limitation and reduced quality of life (QoL). This can lead to socioeconomic burden internationally. In the US, approximately $\$ 70$ billion of the indirect cost is spent in managing LBP. Around $£ 4.1$ and $£ 3.1$ billion are the costs of LBP management in Switzerland [4] and the Netherlands [5], respectively. Interestingly, the current evidence reports the burden of LBP, recurrence of symptom(s) and chronicity worldwide [1, 2]. In Thailand, musculoskeletal disorders are the fourth major health problem, following cardiovascular disease, diabetes, and chronic respiratory disease. Remarkably, LBP is reported as the second most common musculoskeletal problem, following soft tissue disorders [6].

Individuals with non-specific LBP can suffer from physical (e.g., pain and disability) and psychological problems (e.g., pain catastrophising, anxiety, depression and fear-avoidance beliefs) [7]. Consequentially, both components should be addressed to manage individuals with non-specific LBP. In primary care, treatment focused on biopsychosocial problems is an effective management among patients with LBP [8-10]. Thus, assessments in physical and psychological perspectives should be addressed in patients with non-specific LBP.

The Keele STarT Back Screening Tool (SBST) is a simple prognostic screening tool evaluating both physical and psychosocial aspects for LBP patients [11]. It is developed to classify patients with LBP into low, moderate and high risks according to risk for chronicity to help primary care management in the UK [10]. The findings demonstrated that the SBST can be used to reduce disability and cost of management in patients with LBP, approximately $£ 675$ per one patient for socioeconomics burden and approximately $£ 34.39$ for the health services [10]. It can be seen that the SBST can induce appropriate treatment, save time, and decrease the costs of LBP management owing to the stratified care [10].

The SBST is a self-assessment tool with nine items (items 1-4 assessed the physical factors and items 5-9 assessed the psychosocial factors) [10]. The total score ranges from 0 to 9 [10]. Patients with a total score $\leq 3$ will be classified into a low-risk group, whereas patients with a total score $\geq 4$ and a psychosocial subscore $\leq 3$ will be classified into a medium-risk group [10]. A total score $\geq 4$ and a psychosocial subscore $\geq 4$ will be classified into a high-risk group [10]. An appropriate treatment will be designed for patients in each risk group: low-risk group (e.g., self-management and providing information for education), medium-risk group (e.g., physiotherapy to manage pain, functional impairment and disability) and high-risk group (e.g., physiotherapy to manage pain, functional impairment, disability and distress) [10]. Although the SBST has been translated into 38 languages, a Thai version of the SBST is not available to help the primary care management of Thai patients with LBP. Therefore, the aims of this study were to translate and adapt the original version of the SBST into a Thai version and test its psychometric properties in patients with non-specific LBP.

\section{Methods}

This study was divided into two stages: 1) linguistic translation and cross-cultural adaptation of the STarT for Thai patients with LBP and 2) tests of its psychometric properties. All processes were performed in accordance with the Declaration of Helsinki. The permission for translation and cross-cultural adaptation of the STarT was obtained from the Arthritis Research UK Primary Care Centre, United Kingdom, via e-mail. See the following link for further details: https://startback.hfac. keele.ac.uk/training/resources/start-back-translations/.

\section{Stage I: linguistic translation and cross-cultural adaptation}

The translation and cross-cultural adaptation were conducted based on the standard guidelines [12]. Forward translation of the original English version of the STarT into Thai was independently performed by two bilingual translators (Thai and English but Thai first language). The first translator was a musculoskeletal physiotherapist ( $\mathrm{PhD}$ physiotherapy qualification with 12 years of experience) who is familiar with the STarT (provided T1 version). The second translator was an English lecturer (PhD linguistic qualification) and professional translator (provided T2 version). Both translators and the three researchers synthesised the T12 version with a written 
report prior to back translation. Two back translators (PhD linguistic qualification) who were bilingual in Thai and English with no medical background independently translated the T12 version to English without knowledge of the original English version. In the next step, study committee (consisted of the four translators, three researchers, one musculoskeletal physiotherapist and one expert linguistic chair) discussed the original questionnaire, all translational versions and written reports until agreement on the semantic, idiomatic, experiential and conceptual equivalences between the original and targeted versions in order to establish a pre-final version. The pre-final version was tested to assess the clarity of the instruction, comprehensiveness of the items, cultural appropriateness and acceptability using a qualitative cognitive debriefing on 30 patients with LBP who were not included in the data analysis. Comprehension difficulties and further modifications were not reported to the research team. Thus, the Thai version of the SBST was ready for psychometric testing.

\section{Stage II: psychometric properties of the final version Participants}

Potential participants aged 20-70 years old with LBP were recruited from July to December 2019. Individuals with a history of spinal surgery, spinal myelopathy, vertebral fracture, tumours, systemic diseases with possible effect on the musculoskeletal system, pregnancy, and clinically cognitive impairment or who were unable to complete the questionnaire independently were excluded. The participant information sheet and consent form were given to potential participants to provide an opportunity to ask questions, confirm eligibility and obtain written consent. Participants provided written informed consent prior to participation.

\section{Instruments}

Participants were evaluated using the Thai version of all questionnaires.

\section{STarT Back screening tool (SBST)}

The SBST is a fast, simple, valid and reliable screening tool with nine brief items in terms of referred leg pain, comorbid pain, difficulties in walking, difficulties in dressing, fear of physical activity, anxiety, pain catastrophising, depressive mood and overall impact of pain [11]. It was developed to stratify patients into matched treatment pathways based on their prognosis [10]. The SBST response options are dichotomous (agree/disagree) except the ninth item, which is a five-point Likert scale. Prognostic factors covering both physical and psychological aspects are addressed in items 1-4 and items 59, respectively. Patients can be classified into three groups (low, medium and high risk for chronicity).
Those allocated to the high-risk subgroup are at greater risk of persistent disabling LBP symptoms. The total score ranges from 0 to 9 and less than four points is classified as low risk. Patients will be classified as medium or high risk if the total score is higher than three. If the psychological subscore (items 5-9, range from 0 to 5 ) is less than four, the patients will be classified as medium risk; if the sum is equal to or greater than four, patients will be classified as high risk [11].

\section{Visual analogue scale (VAS) for pain intensity}

VAS is a simple and common method with validity and reliability $(\mathrm{ICC}=0.97)$ to assess pain intensity $[13,14]$. It is a 10-cm horizontal line with "no pain" written at the left end point and "worst imaginable pain" at the right end point. Participants were asked to draw a vertical line to mark a point corresponding to the magnitude of their current pain.

\section{Roland-Morris disability questionnaire (RMDQ)}

The RMDQ is a common self-report questionnaire to evaluate LBP disability with 24 (yes/no) items [15]. The total score ranges from 0 to 24 , with a higher score indicating greater disability. The questionnaire was translated and tested for reliability $(n=120$, Cronbach's alpha $=0.83$, range $0.71-0.93$ ) by Jirarattanaphochai et al. for Thai patients with LBP [16].

\section{Fear-avoidance beliefs questionnaire (FABQ)}

The FABQ is a self-report questionnaire with 16 items (each scored 0 to 6 ) covering both work and physical activity [17]. It is a valid and reliable (Cronbach's alpha = 0.88 for work and 0.77 for physical activity, ICC for testreliability $=0.74$ ) tool to assess disability in patients with LBP [17]. The FABQ was translated and cross-culturally adapted into a Thai version and was reported as a valid and reliable tool $(n=129$, Cronbach's alpha range 0.87$\left.0.88, \mathrm{ICC}_{2,1}=0.986\right)[18]$.

\section{Pain Catastrophising scale (PCS)}

The PCS is a valid and reliable self-report tool (Cronbach's alpha $=0.87$ ) with 13 items focusing on thoughts and feelings to measure catastrophising [19]. It contains a three-factor solution: rumination (4 items, Cronbach's alpha $=0.87)$, magnification (3 items, Cronbach's alpha $=0.60)$ and helplessness $(6$ items, Cronbach's alpha $=0.79)$. Each item comprises a five-point Likert scale, ranging from 0 (not at all) to 4 (all the time), resulting in $0-52$ points for a total score. A higher score indicates higher pain catastrophising. The PCS was adapted into Thai with good validity and reliability (Cronbach's alpha $=0.93,0.84$, 0.74 and 0.85 for total score, rumination, magnification and helplessness subscales, respectively) [20]. 


\section{Hospital anxiety and depression scale (HADS)}

The HADS is a valid and reliable tool to assess anxiety (7 items, Cronbach's alpha $=0.83)$ and depression $(7$ items, Cronbach's alpha $=0.82$ ), which is an important prognostic factor for LBP [21, 22]. The total score ranges from 0 to 42, with individual scores ranging from 0 to 3 (a four-point response scale). Higher scores indicate higher levels of anxiety and depression. The HADS was translated and adapted into Thai, demonstrating a valid and reliable tool (Cronbach's alpha $=0.86$ for anxiety and 0.83 for depression) to assess anxiety and depression among Thai patients [23].

\section{EuroQol five-dimensional five-level questionnaire (EQ-5D-}

5L)

The EQ-5D-5L is a valid and reliable self-report QoL questionnaire [24-26]. It is recommended as a useful tool for measuring general health status with five dimensions in terms of mobility, self-care, usual activities, pain/discomfort and anxiety/depression. The score ranges from 0 (dead) to 1 (perfect health). The EQ-5D-5L has been translated into many languages, including Thai and it is a valid and reliable tool $\left(\mathrm{ICC}_{2,1}=0.70\right)[27,28]$.

\section{Statistical analysis}

Descriptive statistics (percentage, means and standard deviation) were used to illustrate participants' demographic characteristics. SPSS statistical package (version 17) was used to analyse validity and reliability as the following.

\section{Content validity}

Content validity was evaluated by the expert committee panel in the translational stage. Floor and ceiling effects ( $>15 \%$ of respondents who achieved the lowest or highest possible SBST-Thai version (SBST-TH) total score were considered) and missing data were identified to evaluate the acceptability [29].

\section{Factor analysis}

Exploratory factor analysis was performed to explore the dimensionality of the SBST-TH using principle component analysis with varimax rotation method. Eigenvalue $\geq 1$ and items of loading $\geq 0.4$ were considered for satisfactory factors, which were named based on included items and their factor loading. Statistical significance $(p<0.05)$ of Bartlett's test of sphericity represents appropriate factor analysis. The Kaiser-Meyer-Olkin (KMO) ranges from 0 to 1 , with higher than 0.5 indicating good factor analysis [30].

\section{Internal consistency}

Internal consistency of the SBST-TH was evaluated using Cronbach's $\alpha$ coefficient. A Cronbach's $\alpha$ value higher than 0.7 was acceptable [29].

\section{Test-retest reliability and agreement}

Test-retest reliability of the SBST-TH was evaluated using intraclass correlation coefficient (ICC) $)_{2,1}$ in 30 participants. The participants were invited to complete the questionnaire twice with an interval of $48 \mathrm{~h}$ to minimise any memory of previous answer and variations in clinical conditions [18]. ICC can range from 0 to 1 . The following criteria were used to interpret the ICC: 0: no reliability, <0.5: unacceptable, $0.5-<0.6$ : poor, $0.6-<0.7$ : questionable, $0.7-<0.8$ : acceptable, $0.8-<0.9$ : good, 20.9: excellent and 1: perfect reliability [31]. The standard error of measurement (SEM = standard deviation of all test scores $\times \sqrt{1-I C C})$ and minimal detectable change $(\mathrm{MDC}=1.96 \times \sqrt{2} \times S E M)$ were calculated to estimate the absolute reliability [32].

\section{Convergent validity}

The correlations between the SBST-TH and VAS (pain intensity), RMDQ, FABQ, PCS, HADS and EQ-5D-5L were examined using Spearman rank correlation coefficient $(r h o)$. The correlations were defined as strong $(r h o \geq 0.5)$, moderate $(0.3 \leq r h o<0.5)$ or weak $(r h o<0.3)$ [33]. According to the previous versions of the SBST [8, 34-37], the scores of the SBST-TH were expected to be significantly correlated to all questionnaires.

\section{Discriminative validity}

Areas under the curve (AUC) were calculated to evaluate discriminative validity between the SBST-TH and the RMDQ, FABQ, PCS and HADS. The cut-offs were defined as follows: RMDQ $\geq 7$ [11], FABQ $\geq 43$ [38], PCS $\geq 20$ [39], and HADS $\geq 8$ [8]. An AUC $=0.5$ indicated "no discrimination", 0.7 to $<0.8$ was considered "acceptable discrimination", 0.8 to $<0.9$ was considered "excellent discrimination" and $\geq 0.9$ indicated "outstanding discrimination" [40]. According to the previous versions of the SBST [8, 41-43], the scores of the SBST-TH were expected to be discriminated to the RMDQ, HADS and PCS.

\section{Results}

A total of 200 patients with non-specific LBP (42 males, 158 females) participated in this study. The demographic and clinical characteristics of the participants are presented in Table 1. 
Table 1 Demographic and clinical characteristics of the participants $(n=200)$

\begin{tabular}{|c|c|c|c|c|}
\hline Variables & $\%(n)$ & Mean \pm SD & Minimum & Maximum \\
\hline Age (year) & & $24.95 \pm 8.75$ & 20.00 & 62.00 \\
\hline \multicolumn{5}{|l|}{ Genders } \\
\hline Female & $79.00(158)$ & & & \\
\hline Male & $21.00(42)$ & & & \\
\hline \multicolumn{5}{|l|}{ Education } \\
\hline No primary school & - & & & \\
\hline Primary school & $1.50(3)$ & & & \\
\hline High school & $91.00(182)$ & & & \\
\hline Bachelor's degree & $6.00(12)$ & & & \\
\hline Master's degree & $1.50(3)$ & & & \\
\hline Doctoral degree & - & & & \\
\hline \multicolumn{5}{|l|}{ Types of low back pain } \\
\hline Acute & $52.00(104)$ & & & \\
\hline Sub-acute & $16.00(32)$ & & & \\
\hline Chronic & $32.00(64)$ & & & \\
\hline \multicolumn{5}{|l|}{ STarT risk group } \\
\hline Low risk & $80.00(160)$ & & & \\
\hline Medium risk & $16.00(32)$ & & & \\
\hline High risk & $4.00(8)$ & & & \\
\hline VAS $(0-100)$ & & $3.11 \pm 1.57$ & 0.40 & 7.40 \\
\hline SBST-TH total score (0-9) & & $2.42 \pm 1.61$ & 0 & 9.00 \\
\hline Physical subscore (0-4) & & $1.17 \pm 1.68$ & 0 & 4.00 \\
\hline Psychological subscore & & $1.24 \pm 1.11$ & 0 & 5.00 \\
\hline RMDQ (0-24) & & $2.44 \pm 2.95$ & 0 & 21.00 \\
\hline FABQ total score $(0-66)$ & & $32.66 \pm 11.85$ & 1.00 & 57.00 \\
\hline FABQ-PA (0-24) & & $13.31 \pm 4.69$ & 0 & 24.00 \\
\hline FABQ-W (0-42) & & $19.35 \pm 8.48$ & 0 & 34.00 \\
\hline PCS total score (0-52) & & $11.21 \pm 8.74$ & 0 & 40.00 \\
\hline Rumination & & $4.63 \pm 3.69$ & 0 & 15.00 \\
\hline Magnification & & $2.90 \pm 2.54$ & 0 & 12.00 \\
\hline Helplessness & & $3.69 \pm 3.59$ & 0 & 18.00 \\
\hline HADS total score (0-42) & & $12.65 \pm 4.95$ & 4.00 & 35.00 \\
\hline Anxiety (0-21) & & $8.29 \pm 2.73$ & 1.00 & 19.00 \\
\hline Depression (0-21) & & $4.36 \pm 2.97$ & 0 & 16.00 \\
\hline EQ-5D-5L index (0-1) & & $0.91 \pm 0.10$ & 0.18 & 1.00 \\
\hline EQ-VAS $(0-100)$ & & $76.81 \pm 14.79$ & 25.00 & 100.00 \\
\hline
\end{tabular}

SD Standard deviation, VAS Visual analogue scale, SBST-TH STarT back screening tool-Thai version, RMDQ Roland-Morris disability questionnaire, FABQ Fearavoidance beliefs questionnaire, $F A B Q-P A$ Fear-avoidance beliefs questionnaire about physical activity, FABQ-W Fear-avoidance beliefs questionnaire about work, $P C S$ Pain catastrophising scale, HADS Hospital anxiety and depression scale, EQ-5D-5L EuroQol five-dimensional five-level questionnaire, EQ-VAS EuroQol-visual analogue scale 
Table 2 Varimax-rotated factor-loading matrix of the SBST-TH

\begin{tabular}{|c|c|c|}
\hline \multirow[t]{2}{*}{ Items } & \multicolumn{2}{|l|}{ Components } \\
\hline & $\begin{array}{l}\text { Factor 1: } \\
\text { Psychological }\end{array}$ & $\begin{array}{l}\text { Factor 2: } \\
\text { Physical }\end{array}$ \\
\hline 1 & & 0.69 \\
\hline 2 & & \\
\hline 3 & 0.41 & $0.62^{\mathrm{a}}$ \\
\hline 4 & 0.67 & \\
\hline 5 & & 0.42 \\
\hline 6 & 0.51 & \\
\hline 7 & 0.47 & $0.48^{\mathrm{a}}$ \\
\hline 8 & 0.80 & \\
\hline 9 & & 0.62 \\
\hline Total variance explained & 27.21 & 12.17 \\
\hline
\end{tabular}

Factors loading $\geq 0.4$ were presented, ${ }^{a}$ factor upon which item loaded most heavily

SBST-TH STarT back screening tool-Thai version

\section{Content validity}

All participants completed all questionnaires with no missing data. No significant floor and ceiling effects were found in the SBST-TH (floor: ceiling $=6.5 \%$ : 0.5\%).

\section{Factor analysis}

The results of factor analyses are presented in Table 2 . Two factors with an eigenvalue $\geq 1$ (39.38\% of the total variance) were extracted. The eigenvalues of two factors (psychological and physical components) were 2.45 and 1.10 , respectively. Bartlett's test of sphericity was 193.19 $(p<0.001)$ and the KMO was 0.70 , demonstrating a good factor analysis of this study.

\section{Internal consistency}

The Cronbach's $\alpha$ values of the SBST-TH total score, physical and psychological subscores were $0.86,0.92$ and 0.76 , respectively. These represent satisfactory internal consistency.

\section{Test-retest reliability}

The mean, standard deviation and ICC for the two testings are presented in Table 3. The ICC of the SBST-TH total $(\mathrm{ICC}=0.73,95 \%$ confidence interval $[\mathrm{CI}]=0.50$
$0.86, p<0.001)$ and psychological subscore $(\mathrm{ICC}=0.79$, 95\% $\mathrm{CI}=0.60-0.89, p<0.001)$ were found to have acceptable reliability.

\section{Agreement}

The SEMs of the SBST-TH and its psychological subscore were 0.74 and 0.58 , respectively. The resultant MDCs were 2.04 and 1.60 , respectively.

\section{Convergent validity}

The correlations between the SBST-TH (including psychological subscore) and VAS (pain intensity), RMDQ, FABQ, PCS, HADS and EQ-5D-5L are presented in Table 4. The SBST-TH total score moderately to strongly correlated to other questionnaires ranging from 0.32 to 0.56 . The moderation correlations were also found between its psychological subscore and other questionnaires, except the FABQ-W $(r h o=0.29,95 \% \mathrm{CI}=$ $0.15-0.41)$, which demonstrated weak correlations.

\section{Discriminative validity}

The AUC of the SBST-TH are presented in Table 5. The range of the SBST-TH total score was from 0.67 $(95 \% \mathrm{CI}=0.55-0.78)$ to $0.85(95 \% \mathrm{CI}=0.72-0.97)$. For the psychological subscore, the AUC range from 0.66 ( 0.55 , $0.77)$ to $0.75(0.52,0.92)$. Only the AUC between the SBST-TH total score and disability (RMDQ) demonstrated excellent discrimination. Other AUC can be considered as acceptable discrimination.

\section{Discussion}

The aims of this study were to translate and crossculturally adapt the original English version of the SBST into Thai and test its psychometric properties. The findings suggest that the SBST-TH had satisfactory reliability and validity in Thai patients with non-specific LBP. The SBST-TH may be easy to understand and use in the Thai population owing to no missing data and no difficulty of collecting data in this study. The findings that no significant floor neither ceiling effects were demonstrated for the SBST-TH suggest a good content validity. Furthermore, the content validity was also verified by the expert committee. Our results were in harmony with

Table 3 Test-retest reliability of the SBST-TH $(n=30)$

\begin{tabular}{|c|c|c|c|c|}
\hline & \multicolumn{2}{|l|}{ Mean \pm SD } & \multirow{2}{*}{$\begin{array}{l}\text { ICC } \\
(95 \% \mathrm{CI})\end{array}$} & \multirow[t]{2}{*}{$p$-value } \\
\hline & First test & Second test & & \\
\hline SBST-TH total & $2.37 \pm 1.27$ & $2.13 \pm 1.28$ & $0.73(0.50-0.86)$ & $<0.001^{*}$ \\
\hline \multicolumn{5}{|l|}{ SBST-TH subscores } \\
\hline Physical component & $1.10 \pm 0.55$ & $1.03 \pm 0.49$ & $0.38(0.02-0.65)$ & $0.02^{*}$ \\
\hline Psychological component & $1.27 \pm 1.02$ & $1.10 \pm 1.00$ & $0.79(0.60-0.89)$ & $<0.001^{*}$ \\
\hline
\end{tabular}


Table 4 Convergent validity

\begin{tabular}{|c|c|c|c|c|}
\hline \multirow[t]{2}{*}{ Questionnaires } & \multicolumn{2}{|l|}{ SBST-TH total score } & \multicolumn{2}{|c|}{ SBST-TH psychological subscore } \\
\hline & $\begin{array}{l}\text { Spearman rank correlation } \\
\text { ( } 95 \% \text { confidence interval) }\end{array}$ & $p$-value & $\begin{array}{l}\text { Spearman rank correlation } \\
\text { (95\% confidence interval) }\end{array}$ & $p$-value \\
\hline VAS pain intensity & $0.45(0.30,0.57)$ & $<0.001^{*}$ & $0.39(0.26,0.50)$ & $<0.001^{*}$ \\
\hline RMDQ & $0.56(0.43,0.69)$ & $<0.001^{*}$ & $0.49(0.36,0.60)$ & $<0.001^{*}$ \\
\hline FABQ total & $0.42(0.29,0.53)$ & $<0.001^{*}$ & $0.33(0.21,0.43)$ & $<0.001^{*}$ \\
\hline FABQ-PA & $0.35(0.23,0.45)$ & $<0.001^{*}$ & $0.32(0.20,0.44)$ & $<0.001^{*}$ \\
\hline FABQ-W & $0.38(0.26,0.51)$ & $<0.001^{*}$ & $0.29(0.15,0.41)$ & $<0.001^{*}$ \\
\hline PCS total & $0.41(0.31,0.55)$ & $<0.001^{*}$ & $0.39(0.26,0.51)$ & $<0.001^{*}$ \\
\hline PSC rumination & $0.37(0.24,0.48)$ & $<0.001^{*}$ & $0.35(0.21,0.48)$ & $<0.001^{*}$ \\
\hline PSC helplessness & $0.37(0.23,0.50)$ & $<0.001^{*}$ & $0.34(0.21,0.47)$ & $<0.001^{*}$ \\
\hline PSC Magnification & $0.38(0.25,0.50)$ & $<0.001^{*}$ & $0.35(0.21,0.48)$ & $<0.001^{*}$ \\
\hline HADS total & $0.38(0.25,0.50)$ & $<0.001^{*}$ & $0.35(0.22,0.45)$ & $<0.001^{*}$ \\
\hline HADS anxiety & $0.34(0.22,0.45)$ & $<0.001^{*}$ & $0.32(0.19,0.44)$ & $<0.001^{*}$ \\
\hline HADS depression & $0.32(0.18,0.44)$ & $<0.001^{*}$ & $0.30(0.15,0.43)$ & $<0.001^{*}$ \\
\hline EQ-5D-5L & $-0.52(-0.61,-0.40)$ & $<0.001^{*}$ & $-0.49(-0.60,-0.38)$ & $<0.001^{*}$ \\
\hline EQ-5D VAS & $-0.41(-0.51,-0.30)$ & $<0.001^{*}$ & $-0.42(-0.54,-0.29)$ & $<0.001^{*}$ \\
\hline
\end{tabular}

SBST-TH STarT back screening tool-Thai version, VAS Visual analogue scale, RMDQ Roland-Morris disability questionnaire, FABQ Fear-avoidance beliefs questionnaire, $F A B Q-P A$ Fear-avoidance beliefs questionnaire about physical activity, FABQ-W Fear-avoidance beliefs questionnaire about work, $P C S$ Pain catastrophising scale, HADS Hospital anxiety and depression scale, EQ-5D-5L EuroQol five-dimensional five-level questionnaire, EQ-VAS EuroQol-visual analogue scale $* p<0.05$

the original version [11] and some adapted versions such as the Chinese [42] and Dutch [5].

Factor analysis identified two factors supporting the original version [11] and Persian version (43\% of the variance demonstrated of the psychosocial component [factor 1] and physical component [factor 2] was 19\%) [35] based on the eigenvalue. The two factors for the SBST-TH explained for $27.20 \%$ of the variance represented psychological component (factor 1) and the physical component (factor 2) was $12.17 \%$. Another factor can be added when only the eigenvalue was considered for factor analysis (eigenvalue $=1.022$ ). The factor can be named "central sensitisation component" (only item 2 and $11.35 \%$ of the variance). Central sensitisation, an increased responsiveness of nociceptors in the central nervous system, is an important prognosis in transitioning to chronicity [44].

High internal consistency of the SBST-TH total score (Cronbach's $\alpha=0.86$ ), physical (Cronbach $\alpha=0.92$ ) and psychosocial subscores (Cronbach's $\alpha=0.76$ ) was found, indicating a homogenous concept of the questionnaire [29]. This was similar to the total score and psychosocial subscore of the original (total score $=0.79$, psychosocial subscore $=0.74)$ [11], Iranian (total score $=0.82$, psychosocial subscore $=0.79$ ) [45] and Persian (total score $=$ 0.83 , psychosocial subscore $=0.81$ ) [35] versions. Unfortunately, previous studies did not provide the internal consistency of the physical subscore. The higher Cronbach's $\alpha$ value of this study may come from the precise and rigorous methodologies using the standardised guidelines of cross-cultural adaptation [12]. Moreover, the ICCs of the SBST-TH total score and its psychosocial subscores were 0.73 and 0.79 , respectively, demonstrating acceptable reliability supporting the original version $(0.73$ for total score and 0.69 for psychosocial subscore) [11] and the Brazilian version (0.79 for total score and 0.76 for psychological subscores) [46]. However, the SBST-TH demonstrated higher ICCs than some previous studies (e.g., Dutch and German versions) $[5,37]$. The variation of the time interval in different

Table 5 Discriminative validity

\begin{tabular}{llll}
\hline Reference standards & Case definition & $\begin{array}{l}\text { AUCs of SBST-TH total score } \\
\mathbf{( 9 5 \% ~ c o n f i d e n c e ~ i n t e r v a l ) ~}\end{array}$ & $\begin{array}{l}\text { AUCs of SBST-TH psychological subscore } \\
\mathbf{( 9 5 \% ~ c o n f i d e n c e ~ i n t e r v a l ) ~}\end{array}$ \\
\hline Disability & $\mathrm{RMDQ} \geq 7$ & $0.85(0.72,0.97)$ & $0.75(0.52,0.92)$ \\
Fear-avoidance beliefs & $\mathrm{FABQ} \geq 43$ & $0.72(0.62,0.81)$ & $0.69(0.60,0.79)$ \\
Catastrophising & $\mathrm{PCS} \geq 20$ & $0.70(0.58,0.81)$ & $0.70(0.58,0.81)$ \\
Depression and anxiety & HADS $\geq 8$ & $0.67(0.55,0.78)$ & $0.66(0.55,0.77)$ \\
\hline
\end{tabular}

AUCS Area under the curves, SBST-TH STarT back screening tool-Thai version, $R M D Q$ Roland-Morris disability questionnaire, $F A B Q$ Fear-avoidance beliefs questionnaire, PCS Pain catastrophising scale, HADS Hospital anxiety and depression scale 
studies may be a factor of the ICC variation. For the previous studies, the average time intervals were 6 days and 10 days, respectively. However, the common intervals were 2 days because a long-time interval may contribute to the patient's clinical status.

The agreement of this study was evaluated by SEM and MDC. The SEM (an error of measurement) of the SBST-TH was 0.74 for the total score and 0.58 for the psychosocial subscore. To the best of our knowledge, only one study (Brazilian version) calculated an SEM higher than in this study (1.90 for the total score) [46]. The small SEMs led to the small MDCs, which were 2.04 and 1.60 for the total score and psychosocial subscore, respectively. These mean change scores equal to or higher than 2.04 for the total score and 1.60 for the psychosocial subscore represent a real change. Unfortunately, the MDC was not calculated from previous studies.

The correlations between the SBST-TH and all reference standard questionnaires to evaluate convergent validity were significant (Table 4). The Spearman's correlation coefficients demonstrated that the overall SBST-TH and its psychosocial subscore moderately to strongly correlated to other questionnaires. The exceptions were the correlations between the psychosocial subscore and FABQ-W, which was weak. Remarkably, the total score was positively strongly correlated with the RMDQ ( $r h o=0.56,95 \% \mathrm{CI}=0.43,0.69$ ), similar to the Japanese [8], German [37], Persian [35], and French [47] versions and negatively strongly correlated with the EQ-5D-5L (rho $=-0.52,95 \% \mathrm{CI}=-0.61,-0.40)$, similar to the Japanese version [8]. These findings suggest a good relationship between the SBST-TH and disability and QoL.

The discriminative validity was demonstrated by AUC for the total score and psychosocial subscore against the case definition by the reference standard questionnaires (Table 5). The highest AUC $(0.85,95 \% \mathrm{CI}=0.72,0.97)$ was observed between the total score of the SBST-TH and disability (RMDQ), reflecting excellent discrimination. This represented that the total score of the SBST$\mathrm{TH}$ can substantially separate cases from physical reference standard. The acceptable discrimination was found in all other AUCs. Interestingly, the AUC of the SBST$\mathrm{TH}$ total score and its psychosocial subscore for the cases defined by psychosocial references (fear-avoidance beliefs, catastrophising and depression and anxiety) were found close to each other. These findings are supported by the original [11] and Japanese [8] versions.

Owing to the results from the convergent and discriminative validity, $\geq 75$ of the a priori hypotheses were confirmed, leading to supporting construct validity of the SBST-TH [29]. However, this study has some limitations. First, the sample size was limited for the factor analysis considering that at least 300 participants would be required for strong factor analysis [48]. Second, the study did not have a question about referred leg pain. This led to a lack of analysis in the case defined by another physical reference for discriminative validity. Third, the sample size of test-retest reliability $(n=30)$ is doubtful and $\geq 50$ participants are recommended according to the COSMIN checklist [49]. Finally, the responsiveness of the SBST-TH was not investigated.

\section{Conclusion}

The translation and adaptation of the Thai version of the SBST were successful. Satisfactory internal consistency, reliability and construct validity of the SBST-TH were demonstrated in this study. Consequentially, the SBST-TH can be applicable in research and clinical settings to classify Thai-speaking clients with non-specific LBP into low, moderate and high risks for chronicity.

\section{Abbreviations}

AUCs: Area Under the Curves; Cl: Confidence Interval; EQ-5D-5L: The EuroQol Five-Dimensional Five-Level questionnaire; EQ-VAS: EuroQol-Visual Analogue Scale; FABQ: Fear-Avoidance Beliefs Questionnaire; FABQ-PA: Fear-Avoidance Beliefs Questionnaire about Physical Activity; FABQ-W: Fear-Avoidance Beliefs Questionnaire about Work; HADS: Hospital Anxiety and Depression Scale; ICC: Intraclass Correlation Coefficient; LBP: Low Back Pain; MDC: Minimal Detectable Change; PCS: Pain Catastrophising Scale; QoL: Quality of Life; RMDQ: Roland-Morris Disability Questionnaire; SBST: STarT Back Screening Tool; SBST-TH: STarT Back Screening Tool - Thai version; SD: Standard Deviation; SEM: Standard Error of Measurement; VAS: Visual Analogue Scale

\section{Acknowledgements}

All authors would like to thank all participants in this study.

\section{Authors' contributions}

TW, NT, NS, TP and NP conceived the idea for the study. TW, NP, NT, NS, TP, IK, DT, RC and WS substantially involved in data collections and interpretations. TW has written and improved the manuscript. All co-authors made a significant intellectual contribution to the manuscript. All authors have read and approved the final manuscript.

\section{Funding}

The authors received no financial support for this study.

\section{Availability of data and materials}

The datasets used and/or analysed during the current study available from the corresponding author on reasonable request.

\section{Declarations}

Ethics approval and consent to participate

The study protocol was approved by the Institutional Review Board of Naresuan University (NU-IRB 0223/62). All processes were performed in accordance with the declaration of Helsinki. All participants provided written informed consent prior to participation.

Consent for publication

Not applicable.

Competing interests

No competing interests. 


\section{Author details}

Exercise and Rehabilitation Sciences Research Unit, Faculty of Allied Health Sciences, Naresuan University, Phitsanulok 65000, Thailand. ${ }^{2}$ Department of Physical Therapy, Faculty of Allied Health Sciences, Naresuan University, Phitsanulok 65000, Thailand. ${ }^{3}$ Department of English Language, Faculty of Humanities, Naresuan University, Phitsanulok 65000, Thailand. ${ }^{4}$ Department of Accounting, Faculty of Business Administration, Chiang Mai University, Chiang Mai 50200, Thailand.

\section{Received: 26 March 2021 Accepted: 7 May 2021}

\section{Published online: 18 May 2021}

\section{References}

1. Vos T, Lim SS, Abbafati C, et al. Global burden of 369 diseases and injuries in 204 countries and territories, 1990-2019: a systematic analysis for the global burden of disease study 2019. Lancet. 2020;396(10258):1204-22.

2. Hartvigsen J, Hancock MJ, Kongsted A, Louw Q, Ferreira ML, Genevay S, et al. What low back pain is and why we need to pay attention. Lancet. 2018;391(10137):2356-67. https://doi.org/10.1016/S0140-6736(18)30480-X.

3. Maher C, Underwood M, Buchbinder R. Non-specific low back pain. Lancet. 2017;389(10070):736-47. https://doi.org/10.1016/S0140-6736(16)30970-9.

4. Tan CIC, Liaw JSC, Jiang B, Pothiawala SE, Li H, Leong MKF. Predicting outcomes of acute low back pain patients in emergency department: a prospective observational cohort study. Medicine. 2018:97(26):e11247. https://doi.org/10.1097/MD.0000000000011247.

5. Bier JD, Ostelo RW, Van Hooff ML, et al. Validity and reproducibility of the STarT Back tool (Dutch version) in patients with low back pain in primary care settings. Phys Ther. 2017;97(5):561-70. https://doi.org/10.1093/ptj/ pzx023.

6. National Statistics of Thailand. The survey of sanitation habofcotp, 2017. Available: http://www.nso.go.th/sites/2014/DocLib13, Accessed 6 Jul 2020. 2017.

7. Field J, Newell D. Relationship between STarT Back screening tool and prognosis for low back pain patients receiving spinal manipulative therapy. Chiropr Man Therap. 2012;20(1):17. https://doi.org/10.1186/2045-709X-20-17.

8. Matsudaira K, Oka H, Kikuchi N, Haga Y, Sawada T, Tanaka S. Psychometric properties of the Japanese version of the STarT back tool in patients with low back pain. PLoS One. 2016;11(3):e0152019. https://doi.org/10.1371/ journal.pone.0152019.

9. Dunn KM, Hestbaek L, Cassidy JD. Low back pain across the life course. Best Pract Res Clin Rheumatol. 2013;27(5):591-600. https://doi.org/10.1016/j. berh.2013.09.007.

10. Hill JC, Whitehurst DG, Lewis M, et al. Comparison of stratified primary care management for low Back pain with current best practice (STarT Back): a randomised controlled trial. Lancet. 2011;378(9802):1560-71. https://doi. org/10.1016/50140-6736(11)60937-9

11. Hill JC, Dunn KM, Lewis M, Mullis R, Main CJ, Foster NE, et al. A primary care back pain screening tool: identifying patient subgroups for initial treatment. Arthritis Rheum. 2008:59(5):632-41. https://doi.org/10.1002/art.23563.

12. Beaton DE, Bombardier C, Guillemin F, Ferraz MB. Guidelines for the process of cross-cultural adaptation of self-report measures. Spine. 2000;25(24):318691. https://doi.org/10.1097/00007632-200012150-00014.

13. Jun $\mathrm{D}$, Zoe M, Johnston V, O'Leary S. Physical risk factors for developing non-specific neck pain in office workers: a systematic review and metaanalysis. Int Arch Occup Environ Health. 2017;90(5):373-410. https://doi. org/10.1007/s00420-017-1205-3

14. Bijur PE, Silver W, Gallagher EJ. Reliability of the visual analog scale for measurement of acute pain. Acad Emerg Med. 2001;8(12):1153-7. https:// doi.org/10.1111/j.1553-2712.2001.tb01132.x.

15. Roland M, Morris R. A study of the natural history of back pain. Part I: development of a reliable and sensitive measure of disability in low-back pain. Spine. 1983;8(2):141-4. https://doi.org/10.1097/00007632-19830300000004.

16. Jirarattanaphochai K, Jung S, Sumananont C, Saengnipanthkul S. Reliability of the Roland-Morris disability questionnaire (Thai version) for the evaluation of low back pain patients. J Med Assoc Thail. 2005;88(3):407-11.

17. Waddell G, Newton M, Henderson I, Somerville D, Main CJ. A fear-avoidance beliefs questionnaire (FABQ) and the role of fear-avoidance beliefs in chronic low back pain and disability. Pain. 1993;52(2):157-68. https://doi. org/10.1016/0304-3959(93)90127-B.
18. Wiangkham T, Phungwattanakul N, Tedsombun P, Kongmee I, Suwanmongkhon W, Chidnok W. Translation, cross-cultural adaptation and psychometric evaluation of the Thai version of the fear-avoidance beliefs questionnaire in patients with non-specific neck pain. Scand J Pain. 2021; 21(2):247-55. https://doi.org/10.1515/sjpain-2020-0116.

19. Sullivan MJ, Bishop SR, Pivik J. The pain catastrophizing scale: development and validation. Psychol Assess. 1995;7(4):524-32. https://doi.org/10.1037/104 0-3590.7.4.524.

20. Youngcharoen P, Aree-Ue S, Saraboon Y. Validation of pain in catastrophizing scale Thai version in older adults with knee osteoarthritis. Innov Aging. 2017;1(Suppl 1):871. https://doi.org/10.1093/geroni/ igx004.3131.

21. Zigmond AS, Snaith RP. The hospital anxiety and depression scale. Acta Psychiatr Scand. 1983;67(6):361-70. https://doi.org/10.1111/j.1600-0447.1983. tb09716.x.

22. Bjelland I, Dahl AA, Haug TT, Neckelmann D. The validity of the hospital anxiety and depression scale: an updated literature review. J Psychosom Res. 2002;52(2):69-77. https://doi.org/10.1016/50022-3999(01)00296-3.

23. Nilchaikovit T, Lotrakul M, Phisansuthideth U. Development of Thai version of hospital anxiety and depression scale in cancer patients. J Psychiatr Assoc Thai. 1996:41(1):18-30.

24. Haywood KL, Garratt AM, Fitzpatrick R. Quality of life in older people: a structured review of generic self-assessed health instruments. Qual Life Res. 2005;14(7):1651-168. https://doi.org/10.1007/s11136-005-1743-0.

25. Janssen M, Pickard AS, Golicki D, et al. Measurement properties of the EQ5D-5L compared to the EQ-5D-3L across eight patient groups: a multicountry study. Qual Life Res. 2013;22(7):1717-27. https://doi.org/10.1007/ s11136-012-0322-4.

26. Bilbao A, García-Pérez L, Arenaza JC, García I, Ariza-Cardiel G, Trujillo-Martín E, et al. Psychometric properties of the EQ-5D-5L in patients with hip or knee osteoarthritis: reliability, validity and responsiveness. Qual Life Res. 2018;27(11):2897-908. https://doi.org/10.1007/s11136-018-1929-x.

27. Kimman M, Vathesatogkit P, Woodward M, Tai ES, Thumboo J, Yamwong S, et al. Validity of the Thai EQ-5D in an occupational population in Thailand. Qual Life Res. 2013;22(6):1499-506. https://doi.org/10.1007/s11136-012-0251-2.

28. Pattanaphesaj J, Thavorncharoensap M. Measurement properties of the EQ5D-5L compared to EQ-5D-3L in the Thai diabetes patients. Health Qual Life Outcomes. 2015;13(1):14. https://doi.org/10.1186/s12955-014-0203-3.

29. Terwee CB, Bot SD, de Boer MR, et al. Quality criteria were proposed for measurement properties of health status questionnaires. J Clin Epidemiol. 2007;60(1):34-42. https://doi.org/10.1016/j.jclinepi.2006.03.012.

30. Tabachnick BG, Fidell LS. Experimental designs using ANOVA. Belmont: Thomson/Brooks/Cole; 2007

31. Koo TK, Li MY. A guideline of selecting and reporting intraclass correlation coefficients for reliability research. J Chiropr Med. 2016;15(2):155-63. https:// doi.org/10.1016/j.jcm.2016.02.012

32. Beckerman $\mathrm{H}$, Roebroeck $\mathrm{M}$, Lankhorst $\mathrm{G}$, et al. Smallest real difference, a link between reproducibility and responsiveness. Qual Life Res. 2001;10(7): 571-8. https://doi.org/10.1023/A:1013138911638.

33. Cohen J. Set correlation and multivariate methods. Statistical Power Analysis for the Behavioral Sciences. 2nd ed. New Jersey: Lawrence Erlbaum Associates; 1988. p. 467-530.

34. Bruyère $\mathrm{O}$, Demoulin $\mathrm{M}$, Beaudart $\mathrm{C}$, Hill JC, Maquet $\mathrm{D}$, Genevay $\mathrm{S}$, et al. Validity and reliability of the French version of the STarT Back screening tool for patients with low back pain. Spine. 2014:39(2):e123-8. https://doi.org/1 0.1097/BRS.0000000000000062.

35. Abedi M, Manshadi FD, Khalkhali M, Mousavi SJ, Baghban AA, Montazeri A, et al. Translation and validation of the Persian version of the STarT Back screening tool in patients with nonspecific low back pain. Man Ther. 2015; 20(6):850-4. https://doi.org/10.1016/j.math.2015.04.006.

36. Aebischer B, Hill JC, Hilfiker R, Karstens S. German translation and crosscultural adaptation of the STarT back screening tool. PLoS One. 2015;10(7): e0132068. https://doi.org/10.1371/journal.pone.0132068.

37. Karstens S, Krug K, Hill JC, Stock C, Steinhaeuser J, Szecsenyi J, et al. Validation of the German version of the STarT-Back tool (STarT-G): a cohort study with patients from primary care practices. BMC Musculoskelet Disord. 2015;16(1):346. https://doi.org/10.1186/s12891-015-0806-9.

38. George SZ, Fritz JM, Childs JD. Investigation of elevated fear-avoidance beliefs for patients with low back pain: a secondary analysis involving patients enrolled in physical therapy clinical trials. J Orthop Sports Phys Ther. 2008;38(2):50-8. https://doi.org/10.2519/jospt.2008.2647. 
39. Sullivan MJ, Stanish WD. Psychologically based occupational rehabilitation: the pain-disability prevention program. Clin J Pain. 2003;19(2):97-104. https://doi.org/10.1097/00002508-200303000-00004.

40. Hosmer DW, Lemeshow S, Sturdivant RX. Area under the ROC curve. Applied logistic regression. $3^{\text {rd }}$ ed. Massachusetts: Wiley; 2013. https://doi. org/10.1002/9781118548387.

41. Morsø L, Kent P, Albert HB, Hill JC, Kongsted A, Manniche C. The predictive and external validity of the STarT Back tool in Danish primary care. Eur Spine J. 2013;22(8):1859-67. https://doi.org/10.1007/s00586-013-2690-z.

42. Luan S, Min Y, Li G, Lin C, Li X, Wu S, et al. Cross-cultural adaptation, reliability, and validity of the Chinese version of the STarT Back screening tool in patients with low back pain. Spine. 2014;39(16):e974-9. https://doi. org/10.1097/BRS.0000000000000413.

43. Morsø L, Kent P, Manniche C, Albert HB. The predictive ability of the STarT Back screening tool in a Danish secondary care setting. Eur Spine J. 2014; 23(1):120-8. https://doi.org/10.1007/s00586-013-2861-y.

44. Arendt-Nielsen L, Morlion B, Perrot S, Dahan A, Dickenson A, Kress HG, et al. Assessment and manifestation of central sensitisation across different chronic pain conditions. Eur J Pain. 2018;22(2):216-41. https://doi.org/10.1 002/ejp.1140.

45. Azimi P, Shahzadi S, Azhari S, Montazeri A. A validation study of the Iranian version of STarT Back screening tool (SBST) in lumbar central canal stenosis patients. J Orthop Sci. 2014;19(2):213-7. https://doi.org/10.1007/s00776-0130506-y.

46. Pilz B, Vasconcelos RA, Marcondes FB, Lodovichi SS, Mello W, Grossi DB. The Brazilian version of STarT Back screening tool-translation, cross-cultural adaptation and reliability. Braz J Phys Ther. 2014;18(5):453-61. https://doi. org/10.1590/bjpt-rbf.2014.0028

47. Bruyère $\mathrm{O}$, Demoulin $M$, Brereton $C$, Humblet $F$, Flynn $D$, Hill JC, et al. Translation validation of a new Back pain screening questionnaire (the STarT Back screening tool) in French. Arch Public Health. 2012;70(1):12. https://doi. org/10.1186/0778-7367-70-12.

48. MacCallum RC, Widaman KF, Zhang $\mathrm{S}$, et al. Sample size in factor analysis. Psychol Methods. 1999;4(1):84-99. https://doi.org/10.1037/1082-989X.4.1.84

49. Mokkink LB, Prinsen CA, Patrick DL, Alonso J, Bouter LM, de Vet HC, et al. COSMIN study design checklist for patient-reported outcome measurement instruments. Amsterdam: Department of Epidemiology and Biostatistics; 2019.

\section{Publisher's Note}

Springer Nature remains neutral with regard to jurisdictional claims in published maps and institutional affiliations.

Ready to submit your research? Choose BMC and benefit from:

- fast, convenient online submission

- thorough peer review by experienced researchers in your field

- rapid publication on acceptance

- support for research data, including large and complex data types

- gold Open Access which fosters wider collaboration and increased citations

- maximum visibility for your research: over $100 \mathrm{M}$ website views per year

At BMC, research is always in progress.

Learn more biomedcentral.com/submissions 